\title{
Construcción, validación y confiabilidad de la escala de adaptación familiar de adultos con trastornos mentales
}

\author{
Mejía-Benavides, Jorge Emmanuel ${ }^{*}$; Álvarez Aguirre, Alicia ${ }^{2}$.
}

\section{RESUMEN}

Introducción: Los trastornos mentales son enfermedades psiquiátricas que se manifiestan por fallas en el proceso de adaptación y que son expresadas mediante conductas anormales, afectando tanto a la persona que la padece como a todos los miembros de su familia. Objetivo: Diseñar y validar un instrumento para medir la adaptación familiar de adultos con trastornos mentales. Metodología: El instrumento fue construido a partir de las fases propuestas por Sánchez y se desarrollaron los reactivos con base en la Clasificación de Resultados de Enfermería (NOC) y, previa revisión de la literatura. El instrumento fue aplicado a 261 integrantes de familias con un miembro con problemas de salud mental de la Región Noreste del Estado de Guanajuato, México. Para la validez del constructo se realizó la extracción de factores con componentes principales y rotación Varimax, con Eigenvalores por arriba de 1 y carga de factor mayor a .40. Resultados: El análisis mostró seis factores con una varianza explicada de 62.84\%, mientras que el coeficiente de confiabilidad de Alfa de Cronbach osciló entre .62 y .83 para las dimensiones y en la escala total .84. Conclusión: El instrumento mostró que es confiable y válido para medir la adaptación familiar.

\section{Palabras clave: Adaptación; familia; salud mental; terminología normalizada de enfermería (DeCS, BIREME).}

\author{
'Maestro en Ciencias de Enfermería, profesor de tiempo completo, Centro Interdisciplinario del Noreste de la Universidad de Guanajuato. Correo: \\ je.mejiabenavides@ugto.mx. ORCID-ID: https://orcid.org/0000-0002-5061-5316 \\ 2Doctor en Ciencias de Enfermería, profesor de tiempo completo, Departamento de Enfermería Clínica, División de Ciencias de la Salud e \\ Ingenierías, Campus Celaya-Salvatierra, Universidad de Guanajuato, México. ORCID-ID: https://orcid.org/0000-0001-5538-7634.
}

Recibido: 06/03/2019

Aceptado: $15 / 06 / 2019$

${ }^{*}$ Autor para correspondencia

\section{Cómo citar este artículo}

Mejía-Benavides JE, Álvarez Aguirre A. Construcción, validación y confiabilidad de la escala de adaptación familiar de adultos con trastornos mentales. SANUS. 2019; (10): 6-19. [Acceso_____ ]; Disponible en: 


\title{
Construção, validação e confiabilidade da escala de adaptação familiar de adultos com transtornos mentais
}

\begin{abstract}
ABSTRATO
Introdução: Os transtornos mentais são transtornos psiquiátricos que se manifestam por falhas no processo de adaptação e são expressos por comportamentos anormais, afetando tanto a pessoa que tem a doença quanto todos os membros de sua família. Objetivo: Desenhar e validar um instrumento para medir a adaptação da família de adultos com transtornos mentais. Metodologia: $O$ instrumento foi desenvolvido a partir das fases propostas por Sanchez e as questões foram desenvolvidas com base na Classificação dos Resultados de Enfermagem (NOC) e na revisão de literatura. O instrumento foi aplicado a 261 familiares de um membro com problemas de saúde mental da Região Nordeste do Estado de Guanajuato, México. Para a validade do construto, extração de fator com componentes principais e rotação Varimax foi realizada com valores de Eigen acima de $1 \mathrm{e}$ carga fatorial maior que 0,40. Resultados: A analise mostrou seis fatores com uma variância explicada de 62,84\%, enquanto o coeficiente de confiabilidade alfa de Cronbach variou entre 0,62 e 0,83 para as dimensões e 0,84 na escala total. Conclusões: O instrumento mostrou que é confiável e válido para medir a adaptação de família.
\end{abstract}

Palavras chave: Adaptação; família; saúde mental; terminologia padronizada de enfermagem (DeCS; BIREME).

\section{INTRODUCCIÓN}

Los Trastornos Mentales (TM) son definidos como "enfermedades psiquiátricas que se manifiestan por fallas en el proceso de adaptación, expresadas principalmente como anormalidades de pensamiento, sentimiento y comportamiento que producen angustia o deterioro de la función"(1) y son causados por factores biológicos, sociales y experiencias adversas (2), asociados a un deterioro de la persona que lo padece ${ }^{(3)}$.

Los TM no solo afectan a la persona que lo padece, sino a todos los integrantes de la familia(4,5), vivan en la misma casa o ya sean independientes. El inicio de un TM en un miembro de la familia es muy traumático, por lo cual es difícil aceptar dicha enfermedad y resultan una serie de cambios en el funcionamiento cotidiano de cada miembro y si los integrantes carecen de recursos para sobrellevar la situación, se producirán múltiples disfunciones de roles ${ }^{(6)}$.

Los TM a menudo llevan a las personas y a sus familias a caer en la pobreza y la desintegración por el costo elevado del tratamiento y el acceso a los servicios necesarios, que en la mayoría de las ocasiones no se encuentran disponibles en todas las ciudades, además muchos de estos servicios no cuentan con los profesionales suficientes, y los módulos de atención sanitaria no poseen redes con más alto nivel de especialización ${ }^{(7)}$.

Además, la carga familiar se va a centrar en las dificultades y desafíos presentados a consecuencia de la enfermedad del familiar y, por ende, acarrean consecuencias negativas tanto al cuidador como a todos los integrantes ${ }^{(8)}$.

Las familias que deben responsabilizarse del cuidado y ayuda del enfermo ${ }^{(9,10)}$ se enfrentan a múltiples situaciones que implican una sobrecarga para sus miembros, los cuales se ven frecuentemente afectados por sentimientos de malestar y sufrimiento(9), esto por muchos factores como lo es la estigmatización del familiar con TM por la sociedad ${ }^{(11)}$.

La evidencia muestra que un entorno familiar adaptativo es indispensable para satisfacer a los miembros de la familia, lo que conlleva a reducir problemas emocionales y de comportamiento de todos los integrantes. Un cuidador familiar requiere un entorno adaptado para funcionar de manera efectiva y ser emocionalmente estable ${ }^{(12)}$.

Las familias pueden no estar preparadas para aceptar la enfermedad mental y pueden entrar en negación, las familias con integrantes con trastornos mentales a menudo tienen una adaptación deficiente ${ }^{(12)}$, por lo tanto, 
un íntegro nivel de adaptación familiar es la clave para un adecuado funcionamiento de la familia el cual contribuye a la conservación de la salud de todos integrantes ${ }^{(10)}$.

Es importante explorar el contexto familiar, la forma en que los integrantes de la familia responden a la necesidad de adaptación a la situación que están viviendo, para así determinar su bienestar individual y familiar.

El constructo de adaptación ha sido estudiado por la teórica Sor Callista Roy, la cual define el concepto como el "proceso y resultado por el cual las personas, con sentimientos y pensamientos utilizan su conciencia y capacidad de elección para crear una integración humana y ambiental" $(13,14)$. Sin embargo, como tal no define la adaptación familiar, a lo cual se realizó un ajuste al concepto general de adaptación integrando las variables familia y trastorno mental y se define como proceso que favorecen los integrantes la aceptación y salir adelante en el cuidado a través de apoyo, situación que requiere de bienestar y ajuste emocional para la seguridad de sí mismos.

La adaptación familiar es el resultado del encuentro apropiado con las necesidades en conjunto de los miembros de una familia y la respuesta eficaz a los requerimientos ${ }^{(12)}$. Las posibilidades de adaptación de la familia van a depender de la salud mental familiar en sus diferentes etapas a lo largo de la vida ${ }^{(15)}$.

En México hasta el momento no se han localizado instrumentos que midan la adaptación familiar con un integrante con trastorno mental, por tal motivo, el propósito del estudio fue diseñar y validar un instrumento para medir la adaptación familiar de adultos con trastornos mentales.

\section{METODOLOGÍA}

Estudio transversal descriptivo, participaron 261 integrantes de familias que cuentan con una persona con algún trastorno mental, por disponibilidad, pertenecientes a la región noreste del estado de Guanajuato, México, durante el mes de agosto y septiembre de 2018.

Los participantes dieron respuesta al instrumento "Escala de adaptación familiar de adultos con trastornos mentales" conformado por 21 preguntas de respuesta tipo Likert de cinco puntos con opciones de nunca a siempre, integrado por seis dimensiones: resiliencia (1, 2, 3 y 4), bienestar (5, 6, 7, 8 y 9), seguridad en el cuidado (10 y 11), ajuste emocional (12, 13, 14 y 15), aceptación del cuidado (16, 17 y 18), apoyo (19, 20 y 21).

Previo a la colecta de datos los participantes dieron su consentimiento mediante firma, respetando lo enunciado en el Reglamento de la Ley General de Salud en Materia de Investigación para la Salud (16). La aplicación del instrumento fue en los hogares de las familias, en un ambiente tranquilo y sin distractores, con suficiente tiempo para responder cada reactivo, así mismo, indicándoles respondieran con la mayor certeza posible y aclarando todas las dudas que surgieron.

El análisis estadístico consistió en análisis factorial y coeficiente de confiabilidad Alfa de Cronbach.

\section{Diseño de instrumento}

El instrumento fue diseñado por docentes pertenecientes al Campus Irapuato-Salamanca y al Campus Celaya-Salvatierra de la Universidad de Guanajuato.

Para lo que se tomó como referencia las fases de construcción de instrumentos propuestas por Sánchez ${ }^{(17)}$ : definir el concepto a medir, clarificar la población objetivo, desarrollar los ítems, evaluar la validez de contenido, desarrollar las instrucciones, probar los ítems, evaluar validez y confiabilidad.

El concepto a medir fue adaptación familiar, la población objetivo se conformó por los integrantes de la familia que tiene un familiar con trastorno mental de la Región Noreste del Estado de Guanajuato, México, en el desarrollo de los ítems se tomó como referencia la Clasificación de Resultados de Enfermería (NOC) $)^{18)}$ 1308: adaptación a la discapacidad física, 1204: equilibrio emocional, 1305: modificación psicosocial: cambio de vida, 2608: resiliencia familiar, 1705: orientación sobre la salud, 2009: estado de comodidad: entorno y 2606: estado de salud de la familia, los cuales fueron modificados para adaptación familiar.

En la validez de contenido se determinó por la evaluación por jueces (tres profesionales de enfermería, dos con grado de Doctor y uno de Maestría de los cuales dos se desempeñan en el ámbito clínico), quienes revisaron la suficiencia, pertinencia y claridad de los reactivos, en lo que se refiere a la evaluación de pertinencia de los reactivos se calculó el índice de concordancia entre observadores e índice de validez de contenido para las evaluaciones de los expertos sobre la pertinencia de cada reactivo, para lo que se les pidió a los expertos que calificaran los reactivos en una escala de dos puntos (de uno=cumple y cero=no cumple). El índice de validez de contenido para el instrumento total es el porcentaje del total de reactivos a los que los expertos dieron la puntuación de uno, el resultado indicó aceptación superior al $80 \%$ de la escala por parte de los expertos.

Posterior a la construcción de los reactivos, se desarrollaron las instrucciones mismas que fueron valoradas por un familiar con integrante con trastorno mental para determinar la comprensión de estas.

Respecto a la evaluación de validez de constructo se realizó la extracción de factores por componentes principales y rotación Varimax, lo que faculta a interpretar la varianza total explicada, con raíces características (Eigenvalores) por arriba de 1 y cargas factoriales mayor a .4019 .

En relación con la confiabilidad, se realizó una evaluación de la consistencia interna del instrumento total mediante el alfa de Cronbach, teniendo como referencia el valor $\alpha=.80$ como aceptable ${ }^{(20)}$.

\section{RESULTADOS}

En la tabla 1 se presenta la estadística descriptiva de los 21 reactivos, el análisis explica que todos los reactivos cuentan 
con suficiente varianza para ser incluidos en el análisis factorial. Las puntuaciones totales de la escala analizada tuvieron un rango de entre 21 y 105 puntos, una media de 60.02 y una $D E=13.59$, con una mediana de 60.71.

La tabla 2 muestra los resultados del análisis de correlación de $r$ de Pearson. Los valores de cada reactivo con la escala total mostraron un ajuste moderado. Por otra parte, las correlaciones fueron positivas y significativas $(p<.001)$

Para el análisis factorial se consideraron los siguientes criterios, la matriz de correlación mostró múltiples valores superiores a .40. Además, la evaluación de la adecuación de la muestra mediante la prueba de Kaiser-MayerOlkin indicó que las variables median factores comunes al obtener un índice de .80. Finalmente, el resultado de la prueba de esfericidad de Barlett fue estadísticamente significativa ( $\left.p<.001, \mathrm{gl}=210, \mathrm{X}^{2}=1799.167\right)$, lo que indicó que se continuaría con el análisis factorial para determinar los factores subyacentes en la matriz de correlación(19,21,22).

Se probó un análisis simple con rotaciones ortogonales, los factores mostraron los mismos niveles de interpretación que el factorial con rotación Varimax. El estudio factorial por componentes principales con rotación Varimax sugirió el análisis con seis factores, que explicaron el $62.84 \%$ del total de la varianza (tabla 3).

Al examinar la matriz de comunalidades se observó que la mayoría son superiores a .40.

En la tabla 4 se presentan los valores del coeficiente de confiabilidad para los seis factores y la escala total, valores considerados aceptables.

\section{DISCUSIÓN}

Debido a que no se cuenta con un instrumento en Latinoamérica que mida las variables estudiadas y ante esta necesidad, se consideró la realización de uno para que el personal de enfermería tenga una base y así poder identificar factores que desencadenen una mala adaptación familiar de las personas con TM, porque como lo menciona Mathew K, et $\mathrm{al}^{(6)}$, se producen alteraciones en los diferentes roles familiares lo que afecta a todos los integrantes ${ }^{(4)}$.

El instrumento será utilizado posteriormente para

Tabla 1. Estadísticos descriptivos

\begin{tabular}{|l|l|l|}
\hline \multicolumn{1}{|c|}{ Reactivo } & \multicolumn{1}{c|}{ Media } & D.E. \\
\hline Ítem 1 & 3.22 & 1.26 \\
\hline Ítem 2 & 2.67 & 1.24 \\
\hline Ítem 3 & 3.67 & 1.09 \\
\hline Ítem 4 & 3.39 & 1.06 \\
\hline Ítem 5 & 3.51 & .97 \\
\hline Ítem 6 & 4.04 & 1.07 \\
\hline Ítem 7 & 3.33 & 1.08 \\
\hline Ítem 8 & 3.41 & 1.00 \\
\hline Ítem 9 & 3.95 & 1.04 \\
\hline Ítem 10 & 3.58 & 1.13 \\
\hline Ítem 11 & 3.45 & 1.12 \\
\hline Ítem 12 & 3.48 & 1.12 \\
\hline Ítem 13 & 2.63 & 1.22 \\
\hline Ítem 14 & 2.41 & 1.17 \\
\hline Ítem 15 & 3.26 & .98 \\
\hline Ítem 16 & 3.15 & 1.21 \\
\hline Ítem 17 & 3.51 & 1.17 \\
\hline Ítem 18 & 3.56 & 1.20 \\
\hline Ítem 19 & 3.78 & 1.08 \\
\hline Ítem 20 & 3.67 & 1.15 \\
\hline Ítem 21 & 3.76 & .97 \\
\hline
\end{tabular}

Fuente: Escala de adaptación familiar de adultos con trastornos mentales 
Tabla 2. Correlaciones de cada reactivo con la escala total

\begin{tabular}{|l|l|l|}
\hline \multicolumn{1}{|c|}{ Reactivo } & \multicolumn{1}{c|}{$\boldsymbol{r}$} & $\boldsymbol{p}$ \\
\hline Ítem 1 & .386 & .001 \\
\hline Ítem 2 & .521 & .001 \\
\hline Ítem 3 & .577 & .001 \\
\hline Ítem 4 & .551 & .001 \\
\hline Ítem 5 & .492 & .001 \\
\hline Ítem 6 & .292 & .001 \\
\hline Ítem 7 & .513 & .001 \\
\hline Ítem 8 & .629 & .001 \\
\hline Ítem 9 & .525 & .001 \\
\hline Ítem 10 & .555 & .001 \\
\hline Ítem 11 & .506 & .001 \\
\hline Ítem 12 & .001 \\
\hline Ítem 13 & .436 & .001 \\
\hline Ítem 14 & .278 & .001 \\
\hline Ítem 15 & .302 & .001 \\
\hline Ítem 16 & .629 & .001 \\
\hline Ítem 17 & .401 & .001 \\
\hline Ítem 18 & .543 & .001 \\
\hline Ítem 19 & .539 & .001 \\
\hline Ítem 20 & .618 & .001 \\
\hline Ítem 21 & .513 & .001 \\
\hline
\end{tabular}

Fuente: Escala de adaptación familiar de adultos con trastornos mentales

futuras investigaciones, para que enfermería pueda tener evidencia sobre el tema e idear estrategias e intervenciones que ayuden a las familias a lograr la adaptación a la circunstancia que se le presenta en ese momento de la vida.

Respecto a la teoría de Roy, colabora a reafirmar lo mencionado en el modo de interdependencia, que se enfoca en las interacciones para dar y recibir afecto, respeto y valor, relacionándose con el contexto social y la interacción ${ }^{(14)}$, siendo esto importante para la resiliencia familiar.

\section{CONCLUSIONES}

Los resultados dan cuenta de un instrumento para medir la adaptación familiar de adultos con TM que es válido y confiable. La importancia reflejada del instrumento para la disciplina de enfermería es poder utilizarlo en la práctica, con el fin de indagar en las estrategias que utilizan los integrantes de las familias con un adulto con TM para adaptarse y de esta manera, generar estrategias de intervención para lograr que los integrantes de las familias se adapten a las situaciones que viven y direccionar el cuidado de enfermería efectivamente.

\section{CONFLICTO DE INTERESES}

Los autores declararon que no existen conflictos de interés potenciales respecto a la investigación, autoría y/o publicación de este artículo.

\section{FINANCIAMIENTO}

Se realizó mediante financiamiento por parte del PFC de la División de Ciencias de la Vida del Campus IrapuatoSalamanca de la Universidad de Guanajuato.

\section{REFERENCIAS BIBLIOGRÁFICAS}

1. National Center for Biotechnology Information. Mental disorders. [Internet]. [acceso 25 Septiembre 2018]. Disponible en: https://www.ncbi.nlm.nih.gov/ mesh/?term=mental+disorder

2. Nathalie A, Díaz D, Ramírez L. Trastorno mental en el contexto carcelario y penitenciario. Carta Comunitaria. 2017; 25(143):77-88. 
Tabla 3. Análisis factorial ajustado a seis factores

\begin{tabular}{|c|c|c|c|c|c|c|}
\hline \multirow[t]{2}{*}{ Reactivos } & \multicolumn{6}{|c|}{ Factores } \\
\hline & 1 & 2 & 3 & 4 & 5 & 6 \\
\hline 24 & .722 & & & & & \\
\hline 23 & .657 & & & & & \\
\hline 22 & .580 & & & & & \\
\hline 12 & .555 & & & & & \\
\hline 11 & .531 & & & & & \\
\hline 13 & & .769 & & & & \\
\hline 14 & & .760 & & & & \\
\hline 19 & & .496 & & & & \\
\hline 18 & & .440 & & & & \\
\hline 20 & & & .883 & & & \\
\hline 21 & & & .851 & & & \\
\hline 5 & & & & .689 & & \\
\hline 4 & & & & .625 & & \\
\hline 6 & & & & .599 & & \\
\hline 9 & & & & .492 & & \\
\hline 1 & & & & & .809 & \\
\hline 2 & & & & & .615 & \\
\hline 3 & & & & & .569 & \\
\hline 16 & & & & & & .833 \\
\hline 17 & & & & & & .774 \\
\hline 15 & & & & & & .539 \\
\hline $\begin{array}{l}\text { \% varianza expli- } \\
\text { cada }\end{array}$ & 12.26 & 12.08 & 10.18 & 10.12 & 9.17 & 9.00 \\
\hline
\end{tabular}

Fuente: Escala de adaptación familiar de adultos con trastornos mentales

Método de extracción: ejes principales con rotación Varimax

Tabla 4. Confiabilidad de los factores

\begin{tabular}{|l|l|l|}
\hline \multicolumn{1}{|c|}{ Factor } & \multicolumn{1}{c|}{ Ítems } & alpha de Crombach \\
\hline F1 & 4 & .66 \\
\hline F2 & 5 & .75 \\
\hline F3 & 2 & .83 \\
\hline F4 & 4 & .68 \\
\hline F5 & 3 & .67 \\
\hline F6 & 3 & .62 \\
\hline Escala Total & 21 & .84 \\
\hline
\end{tabular}

Fuente: Escala de adaptación familiar de adultos con trastornos mentales 
3.Forman-Hoffman V, Batts K, Hedden S, Spagnola K, Bose J. Comorbid mental disorders among adults in the mental health surveillance survey. Annals of Epidemiology [Internet]. 2018 [acceso 13 Marzo 2019]; 28: 468-474. Disponible en: https://doi.org/10.1016/j. annepidem.2018.03.002

4. Crowe A, Lyness KP. Family functioning, coping, and distress in families with serious mental illness. The Family Journal: Counseling and therapy for couples and families [Internet]. 2014 [acceso 25 Septiembre 2018]; 22(2): 186-197. Disponible en: doi:10.1177/1066480713513552

5. Simo B, Bamvita JM, Caron j, Feury MJ. Predictors of mental health service use among individuals with high psychological distress and mental disorders. Psychiatry Research [Internet]. 2018 [acceso 12 Marzo 2019]; 270: 1122-1130. Disponible en: https://doi.org/10.1016/j. psychres.2018.10.019.

6. Mathew K J, Sharma S, Bhattacharjee D. Helping families of persons with mental illness: role of psychiatric social work. Indian J PsySocl Work [Internet]. 2017 [acceso 25 Septiembre 2018]; 8(2): 44-50. Disponible en: doi:10.29120/IJPSW.2017. v8.i2.40

7. Secretaría de Salud. Programa de Acción Específico Salud Mental 2013-2018 [Internet]. 2018 [actualizado 2014; acceso 24 Septiembre 2018]. Disponible en: https://www.gob.mx/ salud/documentos/programa-de-accion-especifico-saludmental-2013-2018

8. Ennis E, Bunting B. Family burden, family health and personal mental health. BMC Public Health [Internet]. 2013 [acceso 25 Septiembre 2018]; 13: 255. Disponible en: doi. org/10.1186/1471-2458-13-255

9. Martín E, Obando D, Sarmiento P. La adherencia familiar en el trastorno mental grave. Aten Primaria [Internet]. 2017 [acceso 25 Septiembre 2018]; 50(9): 519-526. Disponible en: doi.org/10.1016/j.aprim.2017.06.010

10. Casanova-Rodas L, Rascón-Gasca ML, AlcántaraChabelas H, Soriano-Rodríguez A. Apoyo social y funcionalidad familiar en personas con trastorno mental. Salud Ment. 2014;37(5): 443-448.

11. Karim R, Kwan M, Finlay A, Kondalsamy-Chennakesavan S, Toombs M, Nicholson G, et al. Mortality in hospital patients with and without mental disorders: A datalinkage cohort study. Journal of Psychiatric Research. 2019; 111:104-109.

12. Vagharseyyedin S, Gholami M, Hajihoseini M, Esmaeili A. The effect of peer support groups on family adaptation from the perspective of wives of war veterans with posttraumatic stress disorder. Public Health Nurs [Internet]. 2017 [acceso 25 Septiembre 2018]; 36(6): 547-554. Disponible en: doi:10.1111/ phn.13. Roy C. The Roy Adaptation Model. 3rd ed. New Jersey: PEARSON; 2009.

14. Moreno-Fergusson ME. Introducción al Modelo de Adaptación de Callista Roy. En: Muñoz de Rodríguez L, Moreno-Fergusson ME. De la teoría de enfermería a la práctica: Experiencias con proyectos de gestión del cuidado. Bogotá: Universidad de La Sabana; 2016. p. 45-61.

15. Gutkevich EV. Family mental health and adaptation. Journal of the European Psychiatric Association [Internet]. 2010 [acceso 25 Septiembre 2018]; 25(1): 797. Disponible en: doi.org/10.1016/S0924-9338(10)70788-4.

16. Diario Oficial de la Federación. Reglamento de la Ley General de Salud en materia de investigación para la salud [Internet]. DOF.1986 [Acceso 16 Mayo 2018]. Disponible en: http://www.salud.gob.mx/unidades/cdi/nom/compi/rlgsmis. html

17. Sánchez R, Echeverry J. Validación de Escalas de Medición en Salud. Rev. Salud pública. 2004; 6(3):302-318.

18. Moorhead S, Swanson E, Johnson M, Maas M. Clasificación de Resultados de Enfermería (NOC). 6ta ed. España: Elservier; 2018.

19. Mendenhall W, Beaver R, Beaver B. Introducción a la probabilidad y la estadística. 13a ed. México: Cengage Learning; 2010.

20. Polit D, Beck C. Investigación en enfermería. 9a ed. Philadelphia: Wolters Kluwer; 2018.

21. Waltz C, Strickland O, Lenz E. Measurement Nursing and Helath Research. 4ta ed. New York: Springer Publishing Company; 2010.

22. Kellar S, Kelvin E. Munro's Statistical Methods for Helath Care Research. 6ta ed. China: Wolters Kluwer; 2013. 